GUEST EDITORIAL

\title{
Bleeding disorders (part 2)
}

Haemostasis is a physiological process that stops blood loss at the site of injury, while maintaining normal blood flow in the rest of the circulation.

This is accomplished in three physiological steps that occur in rapid sequence: (i) vasoconstriction; (ii) formation of a platelet plug (primary haemostasis); and (iii) stabilisation of clot through crosslinking of insoluble fibrin (secondary haemostasis).

The fibrin mesh that is incorporated into and around the platelet plug serves to strengthen and stabilise the blood clot. Apart from limiting blood loss, the clot allows for vessel and tissue repair. Anticoagulant mechanisms regulate the coagulation system to ensure formation of a clot that is proportional to the injury. A delicate balance between procoagulant and anticoagulant systems is critical for proper haemostasis and for avoiding pathological bleeding or thrombosis. The clot is finally dissolved by the fibrinolytic system, which also performs the function of preventing blood clots in healthy blood vessels.

Bleeding disorders are divided into two broad categories: (i) inherited (discussed in part 1); ; ${ }^{[1]}$ and (ii) acquired (part 2, current issue). ${ }^{[2]}$ This is the second of a two-part CME series on bleeding disorders.

Bleeding manifestations from acquired causes are generally less severe than in the inherited forms and the clinical picture is often dominated by features of the underlying disorder. In clinical practice, however, bleeding is frequently a presenting manifestation of systemic disease and necessitates a multidisciplinary team approach. Investigation of abnormal bleeding requires a comprehensive history, thorough physical examination and systematic laboratory work-up. For the laboratory work-up, an algorithmic approach is incorporated in the article in the current issue as Fig. $1 .^{[2]}$ Basic laboratory tests, viz. international normalised ratio, partial thromboplastin time, full blood count and blood smear microscopy, characterise the nature and extent of the coagulation defect/s and provide a basis for further investigations. It is important to understand the mechanism of haemostatic disturbances that may be applicable to specific disease processes, as interpretation of laboratory findings depends to a large extent on clinical circumstances.
This CME aims to provide an approach to diagnosis and management of acquired bleeding disorders encountered in general practice. Elaborate discussion on the wide variety of bleeding disorders is not possible, but more common conditions such as immune thrombocytopenia are discussed in greater detail. Complex haemostatic derangements may occur in specific clinical scenarios with varying degrees of bleeding and thrombosis. These include cardiopulmonary bypass, trauma, massive blood loss, liver transplantation and complications of pregnancy. Such scenarios are influenced by a multitude of factors with varied aetiologies that merit discussion on discipline-specific platforms, and are therefore beyond the scope of this CME. Select therapeutic modalities may fall outside the scope of general practice, but are nonetheless included to familiarise readers with available therapies.

The authors are indeed grateful for the opportunity to discuss the subject of bleeding disorders, which is an important and dynamic sphere of coagulation.

\section{N Alli}

Department of Molecular Medicine and Haematology, School of Pathology, Faculty of Health Sciences, University of the Witwatersrand, Johannesburg, and National Health Laboratory Service, Johannesburg, South Africa

nazeer.alli@nhls.ac.za

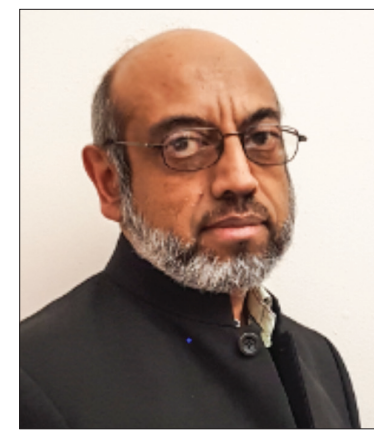

1. Alli N, Vaughan J, Louw S, Schapkaitz E, Mahlangu J. Inherited bleeding disorders. S Afr Med J 2018;108(1):9-15. https://doi.org/10.7196/SAMJ.2018.v108i1.13020

2. Alli N, Vaughan J, Louw S, Moodly S, Patel M. Acquired bleeding disorders. S Afr Med 2018;108(3):159-165. https://doi.org/10.7196/SAMJ.2018.v108i3.13158

S Afr Med J 2018;108(3):158. DOI:10.7196/SAMJ.2018.v108i3.13157 